\title{
Epidemiologie ist mehr als Zählen
}

A uch in diesem Herbst meldet sich die Epidemiologie wieder zu Wort. Die Deutsche Arbeitsgemeinschaft Epidemiologie (DAE) hat vom 9. bis 11. September 2002 ihre 10. Jahrestagung gemeinsam mit der Deutschen Gesellschaft für Medizinische Informatik, Biometrie und Epidemiologie in Berlin abgehalten. Von den dort präsentierten allergologischen Themen dürfen wir Ihnen diesmal drei näher vorstellen, die Ihnen das Spektrum der Public-Health-Forschung beispielhaft näher bringen.

\section{Prävention ganz einfach}

Der Beitrag von Pohlabeln et al. (Seite 442) vom Bremer Institut für Präventionsforschung und Sozialmedizin sowie der Städtischen Kinderklinik in Delmenhorst greift den Bereich Prävention auf. Auf das Thema Prävention waren wir schon an anderer Stelle in diesem Jahr zu sprechen gekommen: zum einen aus methodischer Sicht mit der Frage nach evidenzbasierten Leitlinien zur Allergieprävention und zum anderen mit dem Hinweis auf die vom Bundesministerium für Gesundheit unterstützte Initiative im Rahmen des Aktionsbündnisses Allergieprävention.

Gerade bei Allergien als Beispiel für chronische Erkrankungen, für die bislang keine umfassende kausale Therapie zur Verfügung steht, kommt der Präven-
„Der Prävention kommt bei Allergien eine besondere Bedeutung zu, will man die bevölkerungsbezogene Krankheitslast und die damit verbundenen Kosten wirksam und dauerhaft reduzieren。“ dizinstudenten bis hin zur Schwerpunktsetzung durch Politik und Versicherungsträger bei weitem nicht in ausreichendem Maße berücksichtigt.

Die Ergebnisse der hier vorgestellten Studien an rund 1.000 Müttern zeigen, welche Präventionseffekte durch relativ einfache Maßnahmen der Schulung bevölkerungsbezogen zu erreichen sind. Dies betrifft zum Beispiel Punkte der Ernährung und Allergenexposition. Deutlich werden aber auch Bereiche, in denen ein Schulungsprogramm noch nicht die gewünschten Effekte zeigt. Dies betrifft unter anderem das Stillund Rauchverhalten.

Erschreckend dabei ist die Tatsache, dass rund $60 \%$ der Kinder in den Haushalten gegenüber Passivrauch exponiert sind. Hier erschließt sich ein Präventionspotenzial, das nicht nur aus allergologischer Sicht von hoher Bedeutung ist. Eine frühe Zwischenauswertung zum Zeitpunkt U5 zeigt eine Senkung der Rate von Atopiemanifestationen um 2,5 Prozentpunkte an. Vor dem Hintergrund, dass das Präventionspotenzial noch bei weitem nicht ausgeschöpft ist, macht diese Untersuchung Mut. Gelänge es, die Allergierate in der Bevölkerung nur um einen Prozentpunkt zu redution besondere $\mathrm{Be}$ deutung zu, will man die bevölkerungsbezogene Krankheitslast und die damit verbundenen Kosten wirksam und dauerhaft reduzieren. Auch wenn erfreuliche gesundheitspolitische Ansätze in dieser Richtung zu verzeichnen sind, ist der Bereich Prävention im Gesundheitswesen von der Ausbildung der Me-

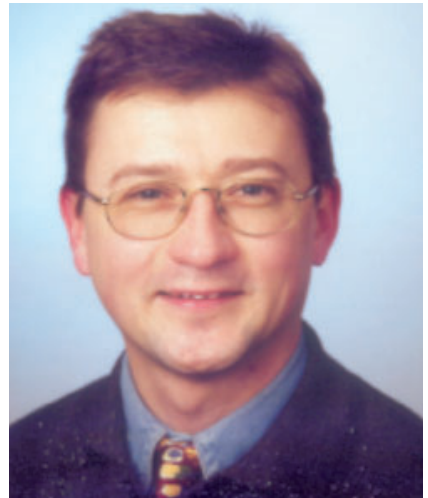

Prof. Dr. Torsten Schäfer, MPH, Sprecher der Arbeitsgruppe Epidemiologie allergischer und dermatologischer Erkrankungen der DAE

Allergologie als klinisch relevanter Endpunkt immer mehr Bedeutung. Dies geschieht mit der Vorstellung, dass besonders bei chronischen und nicht lebensbedrohlichen Erkrankungen am besten der Betroffene selbst über das Ausmaß der Beschwerden und damit auch über einen möglichen Behandlungserfolg berichten kann. Kurz gesagt zielt dieses Konzept darauf ab, dass medizinischkuratives Bemühen in erster Linie nicht Laborwerte, sondern die Befindlichkeit der Betroffenen günstig beeinflussen will.

Das hier vorgestellte Instrument zur Erfassung der Lebensqualität bei Hauterkrankungen ist methodisch gut evaluiert und in der Lage, Unterschiede in der Beeinträchtigung der Lebensqualität zwischen Diagnosegruppen signifikant zu erkennen sowie erkrankungstypische Muster für die Beeinträchtigung in den einzelnen Bereichen der Lebensqualität zu beschreiben. Danach sind Patienten mit chronisch entzündlichen Dermatosen und vor allem solche mit einem atopischen Ekzem von einer besonders starken Beeinträchtigung der Lebensqualität betroffen. Die Detailanalysen zeigen, dass insbesondere $\mathrm{Pa}-$ tienten mit einer Urtikaria über ein ho- 
hes Maß an psychischer Belastung berichten. Die Analysen können uns nicht nur helfen, die einzelnen Beschwerdebilder aus dieser Sicht besser $\mathrm{zu}$ charakterisieren, sondern liefern auch Ansatzpunkte für entsprechende therapeutische Interventionen.

\section{Für wen Alternativverfahren?}

Ein besonders interessantes Gebiet der Versorgungsforschung stellen Ihnen Riehle et al. (Seite 447) in ihrem Beitrag vor. Die Inanspruchnahme von so genannten Alternativverfahren hat offensichtlich in den letzten Jahren stark zugenommen und betrifft in besonderem Maße auch den Bereich der Allergologie.

Wir hatten bereits über wesentliche Determinanten der Inan-
Kurzbeiträge zur DAE-Tagung:

—Allergieprävention durch Frühintervention

— Lebensqualität bei Hauterkrankungen

- Alternativmedizin und Allergien spruchnahme berichtet und stellen Ihnen hier eine psycho-soziale Charakterisierung der Inanspruchnehmer vor. Dazu wurden in einer bevölkerungsbe- zogenen Studie Allergiekranke zu den Themen Lebenszufriedenheit, Kontrollüberzeugung und gesundheitsbezogene Lebensqualität befragt. Im Ergebnis zeigte sich, dass Anwender von Alternativverfahren über eine signifikant höhere Beeinträchtigung ihrer gesundheitsbezogenen Lebensqualität berichten als Allergiker, die bislang keine Alternativverfahren in Anspruch genommen hatten. Dies mag mit zur Motivation beigetragen haben. Die Analyse zur so genannten Kontrollüberzeugung zeigt für den Bereich der fatalistischen Externalität signifikant höhere Werte bei den Nicht-Anwendern von Alternativverfahren, was darauf hindeutet, dass diese in höherem Maße davon überzeugt sind, dass ihr Gesundheitszustand sich nicht

fahren etwas höher als bei Nicht-Anwendern. Insgesamt ließen sich Anwender von Alternativverfahren durch standardisierte psycho-soziale Messinstrumente gut und in Abgrenzung zu Nicht-Anwendern charakterisieren.

Wir hoffen, dass wir Ihnen auch diesmal einige interessante Anregungen aus dem Bereich der Public-HealthForschung vermitteln konnten. Die Arbeitsgruppe Epidemiologie allergischer und dermatologischer Erkrankungen der Deutschen Arbeitsgemeinschaft Epidemiologie steht Ihnen für Fragen gerne offen (www.sozmed.mu-luebeck. de/eade/index.html) und freut sich ebenso über Ihre aktive Mitarbeit.

Mit besten Grüßen bin ich Ihr
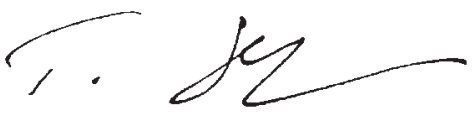
weise liegt die allgemeine wie auch die gesundheitsbezogene Lebenszufriedenheit bei Anwendern von Alternativver-

Prof. Dr. Torsten Schäfer 